\title{
RINGS WITH RESTRICTED MINIMUM CONDITION
}

\author{
AVRAHAM J. ORNSTEIN ${ }^{1}$
}

In what follows, a ring will always mean an associative ring with unit element.

Definition. A ring $R$ is said to satisfy the restricted minimum condition (or to be a RM ring, for short), if for each ideal $A \neq(0)$ in $R$, the ring $R / A$ is right artinian.

In this paper we consider a RM ring, and are furthermore in terested in the case where $R$ itself is not right artinian. The concept of a commutative RM ring was introduced by I. S. Cohen [1], who also proved the following results for a commutative ring $R:$ (a) $R$ is RM iff $R$ is noetherian and every proper prime ideal is maximal. (b) $R$ is RM but not artinian iff $R$ is a noetherian integral domain not a field, in which every proper prime ideal is maximal.

Structure of RM rings. In this section we give a necessary and sufficient condition for a ring to be a $\mathrm{RM}$ ring. It follows by an argument in [1] that in a ring satisfying the ascending chain condition on two-sided ideals, the zero ideal is a finite product of prime ideals. This is true in particular for $\mathrm{RM}$ rings.

LEMma 1. Let $S$ be a ring, such that $S / M$ is a simple artinian ring for each maximal ideal $M$ of $S$. If the zero ideal of $S$ is a finite product of maximal (two-sided) ideals, then either right (left) chain condition implies the other.

Proof. Consider the series $\left(^{*}\right) S=M_{0} \supset M_{1} \supset M_{1} M_{2} \supset \cdots \supset M_{1} M_{2}$ $\cdots M_{k-1} \supset M_{1} M_{2} \cdots M_{k}=(0)$, where $M_{i}, 1 \leqq i \leqq k$ are maximal ideals. The $S$-modules $N_{i}=M_{1} \cdots M_{i-1} / M_{1} \cdots M_{i}$ are completely reducible $S / M_{i}$ modules, and therefore have a composition series in the presence of either chain condition. But $N_{i} \subset S / M_{1} \cdots M_{i}$ inherits the chain condition that is satisfied by $S$. Thus the series $\left({ }^{*}\right)$ can be refined to a composition series of the module $S$.

THEOREM 2. A ring $R$ is RM if and only if

(i) $R$ is a right restricted noetherian ring.

(ii) For each prime ideal $P \neq(0)$ in $R$, the ring $R / P$ is a simple artinian ring.

Received by the editors January 10, 1967.

1 This paper is part of a doctoral thesis submitted to the Graduate School of Rutgers University. The author wishes to express his warmest thanks and appreciation to Professor Carl Faith under whom the thesis was written. 
Proof. Suppose $R$ is a RM ring. Then $R$ satisfies (i) by a theorem of Hopkins and Levitzki [2], [6], and (ii) is satisfied since a prime artinian ring is simple. Conversely, let $I \neq(0)$ be an ideal in $R$, and let $R^{\prime}=R / I$. If $I$ is not prime then by (i), in $R^{\prime}$ the zero ideal is a finite product of prime ideals. If $P^{\prime}$ is any prime ideal in $R^{\prime}$, its inverse image $P$ is a prime ideal in $R$, and $R^{\prime} / P^{\prime} \approx R / P$ is simple artinian by (ii). By Lemma 1, it follows from (i) that $R^{\prime}$ is right artinian.

An ideal $A$ of a ring $R$ will be called essential if $A \cap B \neq 0$ for every ideal $B \neq(0)$.

For later use we prove

Lemma 3. If a ring $R$ contains an essential ideal $A$, so that $R / A$ and $A / B$ are right artinian rings for every ideal $B \neq(0)$ of $R$ contained in $A$, then $R$ is $\mathrm{RM}$.

Proof. Let $I \neq(0)$ be any ideal. Then $(I+A) / I \approx A /(I \cap A)$, and $R /(A+I) \approx R / A /(A+I) / A$ are both right artinian. It follows that so is $R / I$, since $R /(A+I) \approx R / I /(A+I) / I$.

Some properties of RM rings. Let $R$ be a nonprime RM ring. There exists then a finite set of $n>0$ prime ideals (they are necessarily maximal), so that $(0)=P_{1} P_{2} \cdots P_{n}$. We may assume this representation of $(0)$ is not redundant.

THEOREM 4. A nonprime RM ring $R$, has only a finite number of prime ideals, $P_{1}, P_{2}, \cdots P_{n}, n \geqq 1$ which are also the only maximal ideals of $R$. Their intersection $A=\bigcap_{i=1}^{n} P_{i}$ is nilpotent and is precisely the radical $J$. It follows that a nonprime $\mathrm{RM}$ ring has a nilpotent radical.

Proof. Let $P \subset R$ be a prime ideal. Since $(0)=P_{1} \cdots P_{n} \subset P$, it follows that $P_{i} \subset P$ for some $i$, and therefore $P=P_{i}$ by the maximality of $P_{i}$. From $A^{n}=\left(P_{1} \cap \cdots \cap P_{n}\right)^{n} \subset P_{1} \cdots P_{n}=(0)$, we have that $A$ is nilpotent and so $A \subset J$. Since $R / P_{i}$ is simple and right artinian, $\left(R / P_{i}\right) J=(0)$, so that $J \subset P_{i}$ for each $i$ and $J \subset A, J=A$.

CoROllary. A nil ideal in a nonprime RM ring is nilpotent.

As a consequence we have

Theorem 5. A nonprime ring with zero radical, is $\mathrm{RM}$ iff it is right artinian.

Proof. Let $J$ be the radical of the RM ring $R$. Then $J=P_{1} \cap \ldots$ $\cap P_{n}, P_{i}$ maximal ideals. By the Chinese Remainder Theorem [5, p. 28] we have an epimorphism of $R$ on to the direct $\operatorname{sum} \sum_{i=1}^{n} \oplus R / P_{i}$ of the simple artinian rings $R / P_{i}$ with kernel $\bigcap_{i=1}^{n} P_{i}$. Since $J=(0)$, we have $R \approx \sum_{i=1}^{n} \oplus R / P_{i}$. 
Corollary. A nonprime semiprime ring is RM iff it is right artinian.

Proof. Since $J$ is nilpotent, $J=(0)$ in a semiprime ring.

Application to commutative rings. Specializing the results to the commutative case we get a better insight into the structure of a restricted artinian ring $R$ which has zero divisors. Such a ring has only a finite number of prime ideals $P_{1}, P_{2}, \cdots P_{n}$ which are also the only maximal ideals in the ring, and the radical $J(R)$, is equal to their intersection. $R / J(R)$ is then isomorphic to a direct sum of a finite number of fields. In case $R$ has no nilpotent elements, then $R$ itself is such a sum. Conversely, a finite direct sum of fields has trivially the restricted minimum condition. We have

TheOREM 6. A commutative RM ring $R$ which has nonzero zero divisors, has only a finite number of prime ideals, which are also its only maximal ideals. Moreover, $R$ has no nonzero nilpotent elements if and only if $R$ is isomorphic to a finite direct sum of fields.

Semiprimary RM rings. A ring $R$ is called semiprimary if its radical $J$ is nilpotent and $R / J$ is semisimple artinian. As noticed above, a nonprime RM ring is semiprimary. We assume in this section that $R$ is semiprimary and discuss the conditions under which it is RM but not right artinian. It turns out that these properties are determined by the structure of $J$.

Lemma 7. Let $R$ be a semiprimary ring with radical $J$. Then the following conditions are equivalent:

(i) $R$ is right artinian.

(ii) $R / J^{2}$ is right artinian.

(iii) $J / J^{2}$ is a finitely generated right $R$-module.

Proof. Certainly (i) implies (ii), and (ii) implies (iii) by Hopkins and Levitzki's result [2], [6]. That (iii) implies (i) follows from

LEMma 8. Let $R$ be any ring, and $I$ a two-sided ideal in $R$. If the right $R$-module $I / I^{2}$ is finitely generated, then for each integer $k>0$, the $R$-module $I^{k} / I^{k-1}$ is finitely generated.

Proof. Let $x_{1}, x_{2}, \cdots, x_{t}, x_{i} \in I, x_{i} \notin I^{2}$ be a set of representatives of the generators $\bar{x}_{1}, \bar{x}_{2}, \cdots, \bar{x}_{t}$ of $I / I^{2}$. It is easily shown, since $I$ is an ideal, that the finite set of products $x_{i} x_{j}, i, j=1, \cdots, t$ is a set of representatives of generators for $I^{2} / I^{3}$. Repeating this argument, we prove the lemma by induction.

In case $R$ is semiprimary we have $J^{k} / J^{k+1}$ completely reducible 
right $R$-module for each $k$. If furthermore (iii) is assumed, (i) follows since $J$ is nilpotent.

This result was also proved in [7].

THEOREM 9. Let $R$ be a semiprimary $\mathrm{RM}$ ring with radical $J$. The following are equivalent:

(i) $R$ is $\mathrm{RM}$ but not right artinian.

(ii) $J^{2}=(0) . J$ is an essential ideal and is indecomposable as a direct sum of two ideals, and is an infinite direct sum of $R$-isomorphic minimal right ideals. If $J$ contains an ideal $B, J / B$ is a finitely generated $R$ module.

Proof. (ii) implies (i) with the use of Lemma 3. We assume (i) and prove (ii). If $J^{2} \neq(0)$ then $R$ is RM iff $R$ is right artinian by the lemma, and so $J^{2}=(0)$. Certainly $J \neq(0)$, and is completely reducible as a $R / J$ module, therefore also as a $R$-module. We can split the set of minimal summands of $J$ into $m R$-isomorphism classes. The sum of minimal right ideals in each class is an ideal contained in $J$, and $J=A_{1} \oplus \cdots \oplus A_{m}$. Suppose $m>1$ and assume $A_{1}$ is an infinite direct sum of minimal right ideals. Then $A_{1}$ will contain an infinite descending chain $(C)$ of right ideals of $R$. But then $R$ will not be RM, because $R / A_{m}$ say, will contain an infinite descending chain of right ideals corresponding to the chain $(C)$. On the other hand if $A_{1}$, $\cdots, A_{m}$ are all finite direct sums, then the series $R \supset J=A_{1} \oplus \cdots$

$\oplus A_{m} \supset A_{2} \oplus \cdots \oplus A_{m} \supset \cdots \supset A_{m} \supset(0)$ can be refined to a composition series of the right module $R$, and $R$ will be right artinian. If $R$ is to be RM but not right artinian then $m=1$. If $J$ is decomposable as a direct sum of two ideals, then by the same argument as above we can show that $R$ is RM iff it is right artinian. If $J$ is not minimal and contains an ideal $B$, then there exists a right ideal $D$, so that $J=B \oplus D . D$ is completely reducible and by the argument above must be a finite direct sum of minimal ideals. Finally, let $C \neq 0$ be an ideal in $R$ with $J \cap C=(0)$. Then $R / C \supset(J+C) / C \approx J$ is not artinian, in contradiction with (i). This proves that $J$ is essential. (Remark: By the same method one can show that under condition (i), any nonzero ideal is essential, and is not artinian as an $R$-module.)

Prime rings. Applying Theorem 2 we get a condition under which a prime ring will be $\mathrm{RM}$ but not right artinian.

THEOREM 10. Let $R$ be a nonsimple prime ring. Then $R$ will be $\mathrm{RM}$ but not right artinian iff $R$ is right restricted noetherian and for each prime ideal $P \neq(0), R / P$ is a simple artinian ring.

For a prime ring $R$ with nonzero socle, we have 
THEOREM 11. Let $R$ be a prime ring containing a minimal right ideal, the following are equivalent:

(i) $R$ is RM but not right artinian.

(ii) The socle $S$ is an infinite direct sum of minimal right ideals, and $R / S$ is right artinian.

Proof. A prime ring $R$ with a minimal right ideal is right primitive. By $[4$, p. 75$]$, its socle $S$ is a simple ring which is contained in every nonzero ideal of $R$, and is therefore a minimal ideal.

Assume (i). Then $R / S$ is right artinian. But if $S$ should be a finite direct sum of minimal right ideals, this would force $R$ to be right artinian, and we have (ii). Conversely, the first condition of (ii) guarantees that $R$ is not artinian, while the second condition implies that each proper homomorphic image is. For let $A$ be an ideal in $R$, $A \neq(0)$. Then $S \subset A$ and $R / A \approx(R / S) /(A / S)$ is an homomorphic image of a right artinian ring.

Right-sided RA rings. In this section we consider nonartinian right-sided RM rings, i.e. $R$ is not right artinian, but $R / I$ is a right artinian module for every right ideal $(0) \neq I$ of $R$ (RRM rings).

LEMMA 12. Let $R$ be a ring with no right zero divisors and satisfying the ascending chain condition on principal left ideals, then $R$ has the $\mathrm{RRM}$ property on principal right ideals.

Proof. Jacobson [3] proves a similar result on (right and left) principal ideal domains. His method can be used to prove this lemma.

THeOREM 13. A RRM ring is a right Ore domain.

Proof. Let $a \neq 0$ be an element of $R$. Consider the epimorphism $\phi: R \rightarrow a R$ given by $\phi(x)=a x$ for all $x \in R$. The kernel $K$ of $\phi$ is exactly the right annihilator of the element $a$ and is a right ideal of $R$. We have $R / K \approx a R$.

Suppose $K \neq(0)$, then $a R$ is right artinian and therefore by assumption $a R \neq R$. Since $a R \neq(0), R / a R$ is also right artinian, and so is $R$, which is a contradiction. Therefore $K=(0)$, and $R$ is an integral domain.

Suppose there exist two nonzero proper right ideals $I$ and $K$ such that $I \cap K=(0)$. Then $(I+K) / K \approx I$ is right artinian, and by the above argument $R$ is right artinian, which is a contradiction. $R$ is therefore an Ore domain.

Lemma 12 and Theorem 13 imply

CoRollary. $A$ (right and left) principal ideal ring $R$ is RRA if and only if it is a domain. 


\section{BIBLIOGRAPHY}

1. I. S. Cohen, Commutative rings with restricted minimum condition, Duke Math. J. 17 (1950), 27-42.

2. C. Hopkins, Rings with minimal conditions for left ideals, Ann. of Math. (2) 40 (1939), 712-730.

3. N. Jacobson, The theory of rings, Mathematical Surveys, No. 2, Amer. Math. Soc., Providence, R.I., 1943.

4. - Structure of rings, 2nd ed., Amer. Math. Soc. Colloq. Publ., Vol. 37, Amer. Math. Soc., Providence, R.I., 1964.

5. I. Kaplansky, Notes on ring theory, Lecture Notes, Univ. of Chicago, 1956.

6. J. Levitzki, On rings which satisfy the minimum condition for right handed ideals, Compositio Math. 7 (1939), 214-222.

7. B. L. Osofsky, A generalization of quasi-Frobenius rings, (to appear).

RUtGers UNIVERSITY AND

CORNELl UNIVERSITY 